\title{
Performance of the ATLAS RPC detector and Level-1 Muon Barrel trigger at $\sqrt{s}=13 \mathrm{TeV}$
}

\author{
Kunlin $\operatorname{Han}^{a, b}$, on behalf of the ATLAS Collaboration \\ ${ }^{a}$ Department of Modern Physics and State Key Laboratory of Particle Detection and Electronics, \\ University of Science and Technology of China \\ 96 Jinzhai Road, Hefei 230026, China \\ ${ }^{b}$ Laboratoire de Physique des 2 Infinis Irène Joliot Curie \\ 15 Rue Georges Clemenceau, 91400 Orsay, France \\ E-mail: kunlin.han@cern.ch
}

Resistive Plate Chambers (RPCs) are gaseous ionisation detectors that are employed by the Level-1 muon trigger system in the barrel region of the ATLAS muon spectrometer. The Level-1 muon trigger system selects muon candidates that are produced in proton-proton collisions at the Large Hadron Collider (LHC). Muon candidates are associated by the Level-1 system with the correct LHC bunch crossing and with one of the six transverse momentum thresholds. The RPCs are arranged in three concentric double layers and consist of approximately 3700 gas volumes, with a total surface of more than $4000 \mathrm{~m}^{2}$. They operate in a toroidal magnetic field of approximately $0.5 \mathrm{~T}$ and provide up to 6 position measurements along the muon trajectory, with a space-time resolution of about $1 \mathrm{~cm} \times 1 \mathrm{~ns}$. This contribution will discuss performance of the RPC detector and Level-1 Muon Barrel trigger system using proton-proton collision data recorded by the ATLAS experiment in 2018 at a center-of-mass energy of $13 \mathrm{TeV}$.

40th International Conference on High Energy physics - ICHEP2020

July 28 - August 6, 2020

Prague, Czech Republic (virtual meeting) 


\section{Introduction}

ATLAS [1] is a general purpose particle detector recording collisions at the Large Hadron Collider (LHC). The ATLAS muon barrel detector, covering the pseudo-rapidity ${ }^{1}$ range $|\eta|<1.05$, is equipped with the Monitored Drift Tubes and Resistive Plate Chambers (RPC) [2], arranged in three concentric doublet layers at radius $7 \mathrm{~m}, 8 \mathrm{~m}$ and $10 \mathrm{~m}$, operating in a toroidal magnetic field of about $0.5-1.0$ T. The muon barrel geometry is shown in Figure 1a in the $(x, y)$ plane. The Level-1 (L1) muon barrel trigger system [3] uses RPCs for selecting events containing muons in the barrel detector region, produced at $40 \mathrm{MHz}$ collision rate. The muon candidates are selected by the $\mathrm{L} 1$ muon barrel trigger using three low $p_{\mathrm{T}}$ and three high $p_{\mathrm{T}}$ thresholds. Figure $1 \mathrm{~b}$ shows the logic scheme of two algorithms: the low $p_{\mathrm{T}}$ algorithm which requires coincidence between RPC1 and RPC2 doublet layers and the high $p_{\mathrm{T}}$ one which requires a confirmation also in the RPC3 doublet layer.

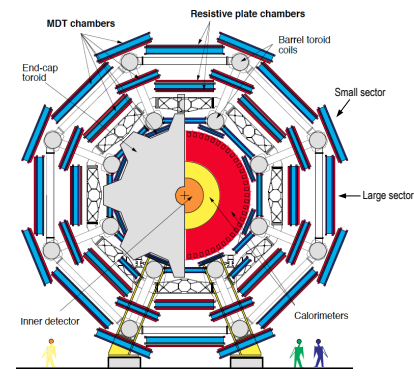

(a)

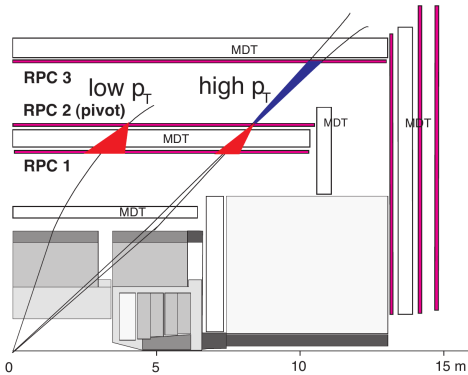

(b)

Figure 1: (a) View of the ATLAS muon spectrometer (MS) barrel detectors in the transverse $(x, y)$ plane. (b) Illustration of L1 muon trigger algorithms in the barrel region [3].

\section{Performance of the RPC detector}

Studies about the detector response and efficiency, the electronics time resolution, the dependence of counting rate and current on the luminosity increase are reported.

\subsection{RPC detector response and efficiency}

The performance of RPC detectors is studied using the Tag\&Probe method [4] on a sample of muons from $Z$ boson production selected with unbiased triggers. Each RPC module can produce several hits in response to the passage of a muon. The average cluster hit multiplicity is shown in Figure 2a, separately for $\eta$ and $\phi$ readout strip panels. Typical muons will produce cluster with single hit in $70 \%$ and $64 \%$ of events for the $\eta$ and $\phi$ readout strip panels, respectively. The average cluster size of all RPC modules is shown in Figure $2 b$. The cluster size of a readout strip panel is computed as the mean value of its cluster hit multiplicity distribution. Some differences in the response of $\eta$ and $\phi$ strip panels are observed. They are mostly due to an asymmetric construction layout and to an additional circuit in the front-end electronics which reverses the polarity of the signal on $\phi$ strips.

The distribution of the detector efficiency is shown in Figure 3a for one ATLAS run. The detector efficiency is defined as the ratio of the number of muons matched to the hits of a panel and

\footnotetext{
${ }^{1}$ The ATLAS experiment uses a right-handed coordinate system. The origin of the system is in the nominal protonproton interaction point, set in the center of the ATLAS detector. The $z$-axis is along the beam line, while the $x y$ plane is the plane perpendicular with respect to the beam line. The positive $x$-axis is defined as pointing from the interaction point to the center of the LHC ring and the positive $y$-axis is defined as pointing upwards.
} 
all probe muons. The gap efficiency is computed by requiring at least one hit in either $\eta$ or $\phi$ strips. Most of the gas gaps have very high efficiency, around 95\%. Figure 3b shows the detector mean efficiency of each run in 2018. The overall detector efficiency is stable across the full data taking and a small step is observed in August due to the retuning of Front-End (FE) electronics thresholds settings.

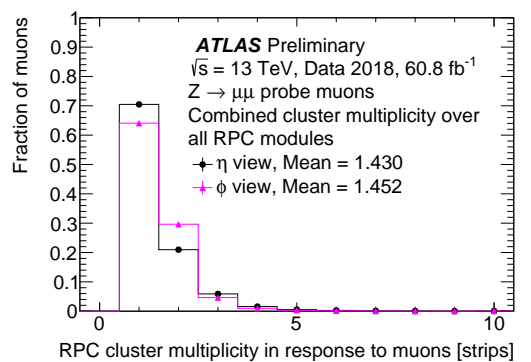

(a)

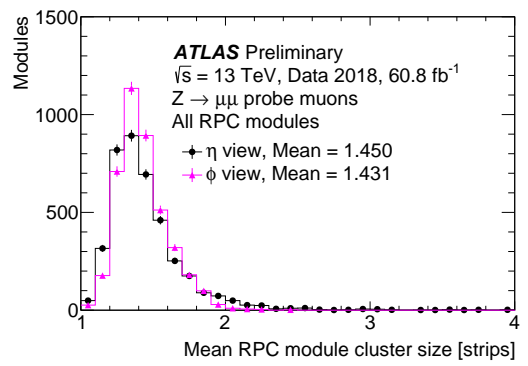

(b)

Figure 2: (a) RPC cluster hit multiplicity distribution averaged over all RPC modules. (b) Mean RPC module cluster size distribution averaged over all RPC modules [5].

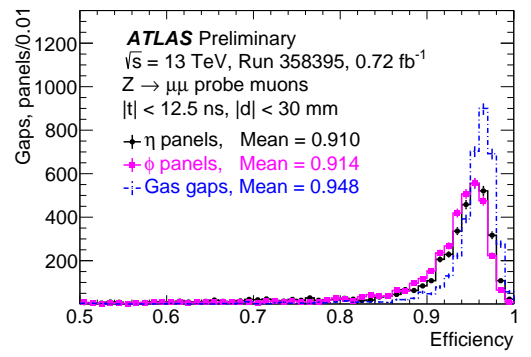

(a)

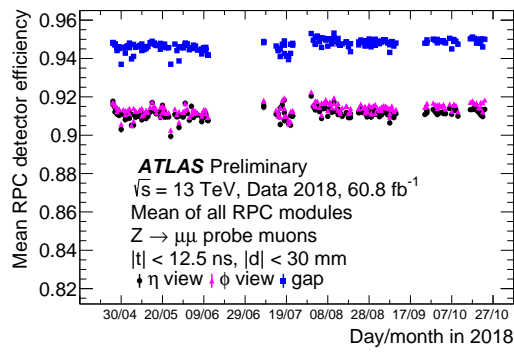

(b)

Figure 3: (a) Distribution of muon detector efficiency for all the active RPC modules. (b) Average muon detection efficiency in each run recorded in 2018 [5]. The selected hits are within \pm 12.5 ns time window and $\pm 30 \mathrm{~mm}$ from the expected muon impact point.

\subsection{RPC detector and electronics time resolution}

The RPC total time resolution $\left(\sigma_{\text {total }}\right)$ is estimated using the hit time difference between detector signals generated by the same muon passing through the two parallel RPC detector layers. Two detector layers are separated by about $20 \mathrm{~mm}$, producing a negligible time-of-flight time. The width of distributions $\left(\sigma_{\text {fit,total }}\right)$ is extracted using a Gaussian function and performing a binned maximum likelihood fit. Figure 4 a shows an example fit on hit time difference distribution for one $\eta-\eta$ strip pair from two layers. This procedure is performed for all strip pairs in both $\eta$ and $\phi$ views as shown in Figure $4 \mathrm{~b}$. The most probable value of total time resolution, defined as $\sigma_{\text {fit,total }} / \sqrt{2}$, is about $1.5 \mathrm{~ns}$. The difference of total time resolution comes from the different cluster size composition between $\eta$ and $\phi$ panels, as already highlighted in Figure 2 .

The RPC total time resolution consists of intrinsic detector resolution and a component related to FE electronics, called simply electronics time resolution. The electronics component ( $\sigma_{\text {electronics }}$ ) is estimated using the hit time difference of signals recorded by a pair of $\eta-\phi$ strips observing the same avalanche in the same detector layer. A maximum likelihood fit is performed to extract the width of distributions using a Gaussian function. All possible $\eta-\phi$ strip pairs are considered to compute statistically weighted average values, shown in Figure 5a. The intrinsic 
component of the time resolution is extracted using the formula: $\sqrt{\sigma_{\text {total }}^{2}-\sigma_{\text {electronics }}^{2}}$ and shown in Figure 5b.

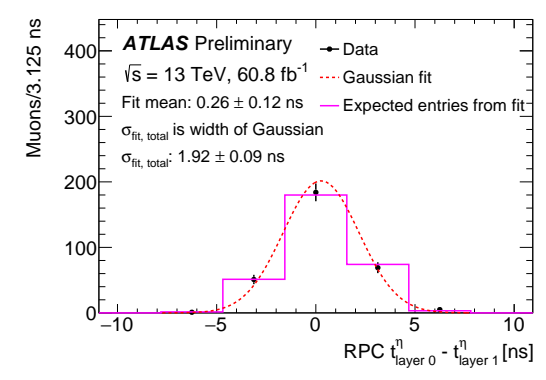

(a)

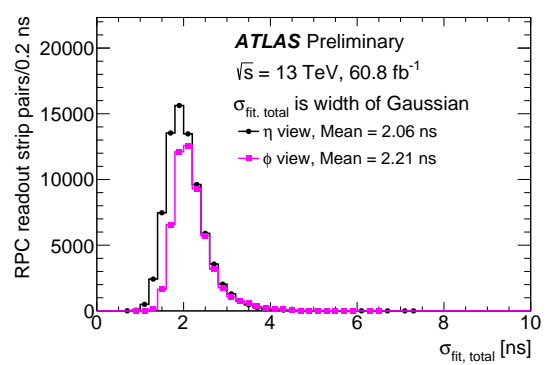

(b)

Figure 4: (a) Time difference distribution between signals generated by the same muons in two $\eta$ strips in two parallel RPC detector layers. (b) Gaussian width of total time difference distribution for the whole RPC system [5].

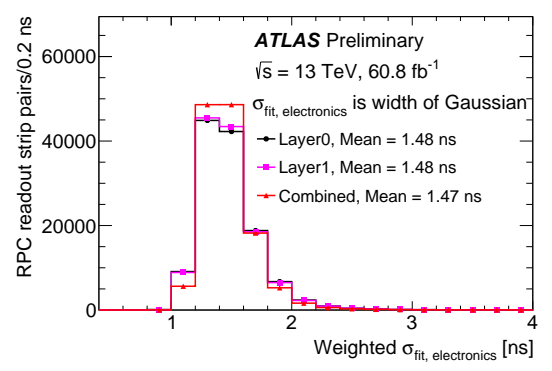

(a)

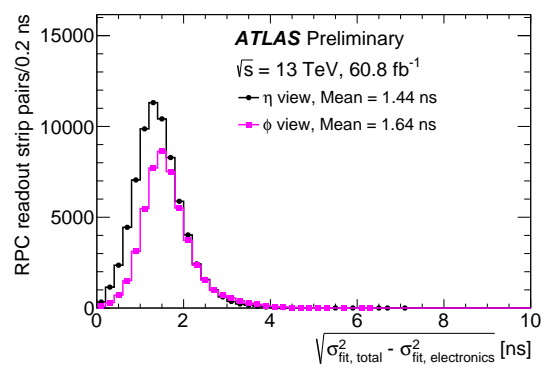

(b)

Figure 5: Distributions of the electronic component (a) and intrinsic component (b) of the time resolution for the whole RPC system [5].

\subsection{RPC counting rates and currents}

The ATLAS RPC counting rate is dominated by secondary particles, mostly photons and neutrons produced in the interactions with detector materials of primary particles. An unbiased sample of average LHC collisions is selected using detector hits in a time window preceding the bunch crossing which contains triggered muons from a hard scattering event. RPC counting rates are computed as a function of instantaneous luminosity for three representative RPC panels at different radii, as shown in Figure 6a. As expected, hit rates depend linearly on the instantaneous luminosity. Smaller hit rates are observed in panels located further away from the interaction point since the flux density of ionising particle decreases at large radii. Figure $6 \mathrm{~b}$ shows the RPC counting rate as a function of $\eta$ and $\phi$ position of the innermost doublet (RPC1) layer at an instantaneous luminosity of $1.8 \times 10^{34} \mathrm{~cm}^{-2} \mathrm{~s}^{-1}$. Higher hit rates are expected in the panels of the forward region due to higher flux density.

In order to check the stability of the detector at various LHC running conditions, the RPC current density is measured as a function of the instantaneous luminosity for all RPC modules. Figure $7 \mathrm{a}$ shows the measured current densities as a function of the instantaneous luminosity for several representative modules in the same $\phi$ sector and at different $\eta$ positions. The expected linear dependency of currents is observed for luminosities up to $2.1 \times 10^{34} \mathrm{~cm}^{-2} \mathrm{~s}^{-1}$. The current density decreases at larger radii due to reduced particle flux density. 
The avalanche charge is another key parameter that characterises the performance of the RPC detector. Its value is given by the gain of the electron amplification process inside the gas and depends on the electric field across the gas gap, gas mixture and gas gap width. The effective avalanche charge is computed using the ratio of gas gap current and counting rate. The distribution of effective avalanche charge is shown in Figure $7 \mathrm{~b}$ for all ATLAS RPC modules. The mean avalanche charge of $30.4 \mathrm{pC}$ is in good agreement with previous measurement performed using a $\gamma$ irradiation source [7].

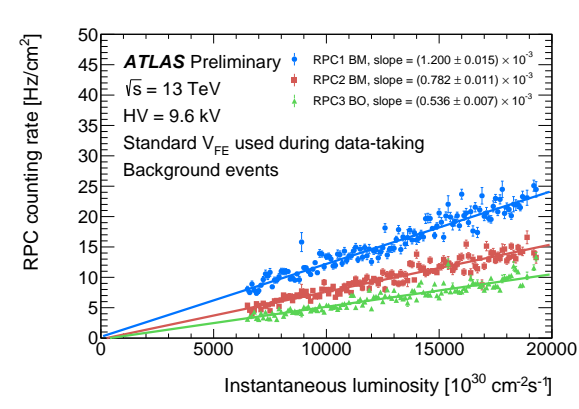

(a)

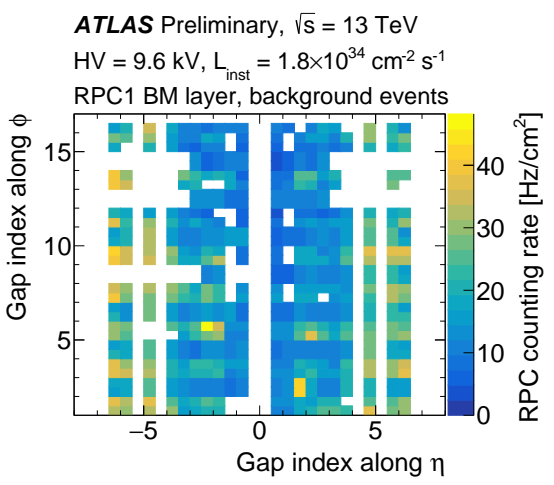

(b)

Figure 6: (a) RPC counting rate as a function of the instantaneous luminosity for RPC panels. (b) Counting rate as a function of $\eta$ and $\phi$ positions of RPC1 layer [5].

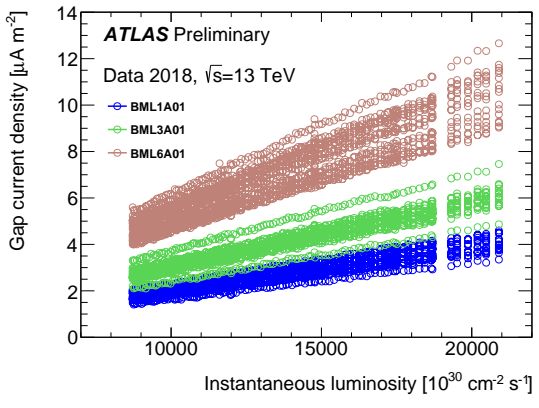

(a)

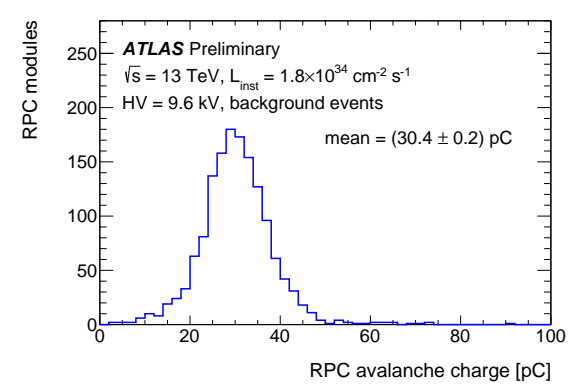

(b)

Figure 7: (a) RPC current density as a function of instantaneous luminosity for RPC modules at different $\eta$ positions [6]. (b) Averaged RPC avalanche charge distribution per single detector hit. [5]

\section{Performance of Level-1 muon barrel trigger}

The association of a L1 muon trigger candidate to the correct bunch crossing (BC) requires accurate timing calibration of RPC readout electronics. These calibrations correct for different signal propagation of different RPC channels and are performed in steps of $3.125 \mathrm{~ns}$. The overall fraction of trigger hits associated to the correct $\mathrm{BC}$ is measured to be about $96 \%$ and is observed to be stable during the data taking period of 2018.

Trigger efficiency is one of the key parameters of the L1 muon barrel trigger system and it is monitored to be stable during the data taking period using probe muons from $\mathrm{Z}$ boson decay. Figure 8a shows the trigger efficiency as a function of offline muon $p_{\mathrm{T}}$ for six $p_{\mathrm{T}}$ thresholds. The trigger efficiency plateau value in each run is plotted as a function of time in Figure 8b. Taking into account geometrical acceptance effects, the overall efficiency to detect muon candidates is about $76.5 \%$ for low $p_{\mathrm{T}}$ thresholds and about $70 \%$ for high $p_{\mathrm{T}}$ thresholds with good stability. 


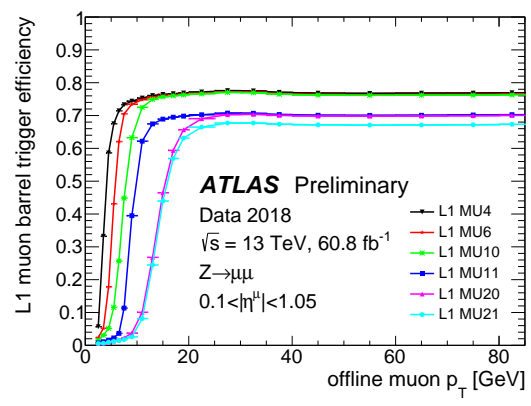

(a)

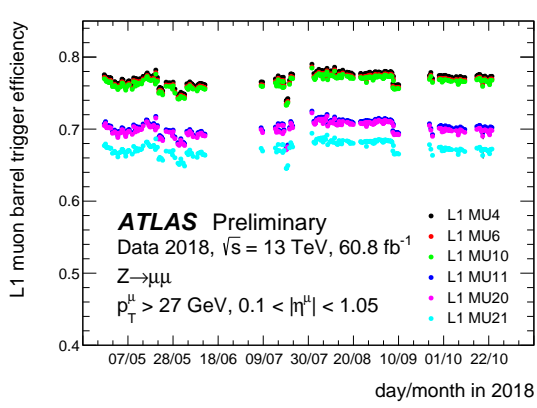

(b)

Figure 8: (a) L1 muon barrel trigger efficiency as a function of the offline muon transverse momentum for several values of trigger thresholds. (b) Plateau values of trigger efficiency in each run recorded in 2018 for several values of trigger thresholds [9].

\section{Conclusion}

The performance of the RPC detector and L1 muon barrel trigger are measured using protonproton collision data recorded by the ATLAS experiment at a center-of-mass of $13 \mathrm{TeV}$ in 2018 at the LHC. The average RPC cluster size is approximately 1.5 strip wide. Most of the RPC modules show high efficiency, above $90 \%$. The efficiency is observed to be stable during the 2018 data taking period. The intrinsic time resolution of RPC detector is measured to be about $1 \mathrm{~ns}$. RPC counting rate and current densities scale linearly as a function of instantaneous luminosity, up to $2.1 \times 10^{34}$ $\mathrm{cm}^{-2} \mathrm{~s}^{-1}$. The effective avalanche charge is measured to be about $30 \mathrm{pC}$, in good agreement with previous results. Good performance and stability are observed for L1 muon barrel trigger efficiency. In summary, The ATLAS RPC detector and L1 muon trigger show excellent performance since their completion in 2008.

\section{References}

[1] ATLAS Collaboration, The ATLAS experiment at the CERN Large Hadron Collider, JINST 3 (2008) S08003.

[2] R. Santonico and R. Cardarelli, Development of Resistive Plate Counters, Nucl. Instrum. Meth. 187 (1981) 377 (cit. on p. 2).

[3] F. Anulli et al., The Level-1 Trigger Muon Barrel System of the ATLAS experiment at CERN, JINST 4 (2009) P04010.

[4] ATLAS Collaboration, Muon reconstruction performance of the ATLAS detector in proton-proton at $\sqrt{s}=13 \mathrm{TeV}$, Eur. Phys. J. C 76 (2016) 292, arXiv: 1603.05598 [hep-ex].

[5] ATLAS Collaboration, RPC Detector Performance in 2018 with proton-proton collisions at $13 \mathrm{TeV}$, https://atlas.web.cern.ch/Atlas/GROUPS/MUON/PLOTS/MDET-2020-01.

[6] ATLAS Collaboration Studies of gas gaps current density in the ATLAS RPC detector during 2018 data taking at Large Hadron Collider https://atlas.web.cern.ch/Atlas/GROUPS/MUON/PLOTS/MUON-2020-001.

[7] G. Aielli et al., An RPC gamma irradiation test, Nucl. Instrum. Meth. A 456(2000) 82, ed. by G. Grancagnolo, G. Iaselli, S. Ratti and R. Santonico (cit. on p. 33) 
[8] ATLAS Collaboration Level 1 Barrel Muon trigger and RPC performance in 2015, https://twiki.cern.ch/twiki/bin/view/AtlasPublic/L1MuonTriggerPublicResults.

[9] ATLAS Collaboration, Level 1 muon barrel trigger performance in 2018, https://twiki.cern.ch/twiki/bin/view/AtlasPublic/L1MuonTriggerPublicResults. 